\title{
Future Observations of Transits and Light Curves from Space
}

\author{
Charles A. Beichman ${ }^{1}$, Tom Greene ${ }^{2}$, and John Krist ${ }^{3}$ \\ ${ }^{1}$ Michelson Science Center, California Institute of Technology, 770 S. Wilson Ave., \\ Pasadena, CA 91125 \\ email: chas@ipac.caltech.edu; ${ }^{2}$ Ames Research Center; ${ }^{3}$ Jet Propulsion Laboratory
}

\begin{abstract}
A variety of new observational opportunities have made transit and more generally light curve analysis central to the study of exoplanets. Talks at this IAU 253 Symposium have dramatically highlighted the measurement of the radius, density, atmospheric composition and atmospheric thermal structure, presently for relatively large, hot planets, but soon for smaller planets orbiting further from their host stars. On-going and future space observations will play a key role in the detection and characterization of these planetary systems. After a brief review, I focus on two topics: the need for a sensitive all-sky survey for planets transiting the brightest, closest stars and the follow-up opportunities afforded by the James Webb Space Telescope (JWST).
\end{abstract}

\section{Introduction}

Despite violating the physics teacher's admonition against subtracting two big numbers to measure a smaller value, transits have proven to be a remarkably robust method by which to study exoplanets.

The importance of transits of "hot Jupiters" is due to their easy detection from the ground and the power of precision follow-up from space. The advantages of space for transit observations are multi-fold:

- Straightforward technology compared with the requirements for angular separation of star-planet systems demanded by TPF-C and TPF-I/Darwin (Beichman et al. 2007).

- Highly accurate photometry due to a stable instrumental Point Spread Function (PSF), the lack of seeing effects, and a low, stable sky background.

- Continuous, broad wavelength coverage and sensitive infrared operation.

- Continuous, long-term coverage subject only to orbital, spacecraft, and Time Allocation Committee constraints.

Of course, there are a few drawbacks to space observations:

- Ultra-precision photometry is hard even in space due to effects of Moon- and Earthshine, cosmic rays and other radiation effects.

- Limited telemetry that results in having to analyze postage stamps not full images.

- Long, expensive development for space missions.

Table 1 summarizes some of the ongoing, planned and proposed projects dedicated to or capable of exoplanet observations. Many of these are treated in their own papers in this volume and will not be discussed further. The rest of this paper focuses on two topics: surveys for a broader range of spectral types and for brighter stars, and follow-up observations for detailed characterization. 
Table 1. Transit Opportunities

\begin{tabular}{|c|c|c|c|c|c|}
\hline Mission & Survey & $\begin{array}{c}\text { Primary } \\
\text { Transit }\end{array}$ & $\begin{array}{c}\text { Primary } \\
\text { Spectroscopy }\end{array}$ & $\begin{array}{l}\text { Secondary } \\
\text { Eclipse }\end{array}$ & $\begin{array}{c}\text { Secondary } \\
\text { Spectroscopy }\end{array}$ \\
\hline \multicolumn{6}{|c|}{ On-going Missions } \\
\hline $\begin{array}{l}\text { Spitzer (Cold) } \\
\text { Spitzer (Warm) } \\
\text { HST (+SM4) } \\
\text { MOST } \\
\text { EPOCH }\end{array}$ & $\begin{array}{c}\quad \mathrm{N} / \mathrm{A} \\
<1 \text { sq. deg. } \\
<1 \text { sq. deg. } \\
\text { N } / \mathrm{A} \\
\text { N/A }\end{array}$ & $\begin{array}{l}\text { IRAC } \\
\text { IRAC } \\
\text { STIS, NICA } \\
\text { Excellent } \\
\text { Excellent }\end{array}$ & $\begin{array}{l}\text { IRS } \\
\text { N/A } \\
\text { IOS, } \mathrm{ACS}, \text { STIS } \\
\mathrm{N} / \mathrm{A} \\
\mathrm{N} / \mathrm{A}\end{array}$ & $\begin{array}{c}\text { IRAC/MIPS } \\
\text { IRAC } \\
\text { NICMOS } \\
\text { Limits } \\
\text { Possible }\end{array}$ & $\begin{array}{c}\text { IRS } \\
\text { N/A } \\
\text { NICMOS } \\
\text { N/A } \\
\text { N/A }\end{array}$ \\
\hline \multicolumn{6}{|c|}{ Planned Missions } \\
\hline $\begin{array}{l}\text { Kepler } \\
\text { JWST }\end{array}$ & $\begin{array}{c}100 \text { sq. deg. } \\
\text { N/A }\end{array}$ & $\begin{array}{c}\text { Excellent } \\
\text { NIRSp }\end{array}$ & $\begin{array}{c}\mathrm{N} / \mathrm{A} \\
\mathrm{ec}, \mathrm{NIRCam}\end{array}$ & $\begin{array}{c}\text { Good } \\
\text { NIRSpec, N }\end{array}$ & $\begin{array}{c}\mathrm{N} / \mathrm{A} \\
\text { IRCam,MIRI }\end{array}$ \\
\hline $\begin{array}{l}\text { PLATO } \\
\text { TESS } \\
\text { ASTrO } \\
\text { TRACER } \\
\text { THESIS }\end{array}$ & $\begin{array}{c}550 \text { sq. deg. } \\
\text { Visible, All Sky } \\
\text { Near-IR, All Sky } \\
\text { N/A } \\
\text { N/A }\end{array}$ & $\begin{array}{c}\text { Excellent } \\
\text { Excellent } \\
\text { Excellent } \\
\text { Excellent } \\
\text { Good }\end{array}$ & $\begin{array}{c}\mathrm{N} / \mathrm{A} \\
\mathrm{N} / \mathrm{A} \\
\mathrm{N} / \mathrm{A} \\
\text { Excellent } \\
\text { Good }\end{array}$ & $\begin{array}{c}\text { Good } \\
\text { N/A } \\
\text { N/A } \\
\text { Good } \\
\text { Excellent }\end{array}$ & $\begin{array}{c}\mathrm{N} / \mathrm{A} \\
\mathrm{N} / \mathrm{A} \\
\mathrm{N} / \mathrm{A} \\
\text { Good } \\
\text { Excellent }\end{array}$ \\
\hline
\end{tabular}

\section{Future Space-based Surveys}

With the zeroth generation (MOST) and first generation missions (CoRoT and Kepler) either taking transit data or soon to be launched (Green et al. 2003; Rowe et al.; Baglin et al., Borucki et al., this volume), a number of groups are considering second generation transit missions. With the difficulty of follow-up directly related to the distance (brightness) of the host stars, it is useful to define a metric for transit surveys, e. g. the distance needed to provide a sample of at least 1,000 solar type stars (F5-K5, IV-V) which is approximately $25 \mathrm{pc}$ in the solar neighborhood. The distance to the average transiting host star depends on the alignment probability, $\beta(\sim 1 \%)$, and the solid angle, $\Omega$, of the survey, $D=25 \frac{3}{4} \beta^{-1 / 3}(41,250 \mathrm{sq} \text {. deg. } / \Omega)^{1 / 3}$ pc. Even for an all-sky survey the average transiting systems will be $\sim 25$ pc away because of the alignment requirement (Table 2). On-going space-based surveys of 40-100 sq. deg. (CoRoT and Kepler) will have host stars hundreds of parsecs away with correspondingly faint magnitudes $(\mathrm{V} \sim 12-13$ mag), although looking down a spiral arm improves the average distance compared to a spherical distribution of stars. Thus, an obvious direction for future space missions is to expand the field of view to encompass more and brighter stars. Another target for new surveys is $M$ stars, which offer, with their small radius and low luminosity, an enhanced transit signal and a habitable zone located close to the star for enhanced detectability of "warm" planets (J. Irwin et al., this volume).

\subsection{PLATO}

The European Space Agency (ESA) has selected PLATO for Phase A assessment as a candidate for a Cosmic Vision mission with a launch around 2017. PLATO utilizes 28 small $(0.1 \mathrm{~m})$ telescopes viewing the same $550 \mathrm{sq}$. deg. field with high sensitivity for both transits and asteroseismology of host stars (Roxburgh et al. 2007). With five times the field of view of Kepler, PLATO will observe roughly 4 times as many stars as Kepler to same SNR and will measure 75,000 dwarfs brighter than $\mathrm{V}<12$ mag with $\mathrm{SNR}>37,000$. The typical PLATO transit host will be 1-2 mag brighter than Kepler's. In addition to transit and light curve observations PLATO will provide asteroseismology data on host 
Table 2. Transit Surveys

\begin{tabular}{lcccccc}
\hline \multirow{2}{*}{ Survey Property } & Ground & \multicolumn{5}{c}{ Space-Based } \\
& Based & CoRoT & Kepler & PLATO & TESS & ASTrO \\
\hline$\Omega$ (sq. deg.) & $10^{2} 10^{4}$ & 40 & 100 & 550 & 40,000 & $>25,000$ \\
$\lambda$ & Vis & Vis & Vis & Vis & Vis & Near-IR \\
\# Stars & $10^{3}-10^{5}$ & $10^{5}$ & $10^{5}$ & $10^{5}$ & $2.5 \times 10^{6}$ & $2 \times 10^{6}$ \\
Brightness (mag) & $6-15$ & $11-15$ & $10-14$ & $8-14$ & $4-13.5$ & $4-13.5(\mathrm{~J})$ \\
Precision (ppm) & 2,000 & 50 & $25,1 \mathrm{hr}$ & $25,1 \mathrm{hr}$ & $77,1 \mathrm{hr}$ & $50-100,1 \mathrm{hr}$ \\
& & & $\mathrm{V}=11 \mathrm{mag}$ & $\mathrm{V}=11 \mathrm{mag}$ & $\mathrm{I}=9 \mathrm{mag}$ & $\mathrm{J}=9 \mathrm{mag}$ \\
Avg. FGK star Dist. (pc) & $25-1,000$ & 850 & 650 & 350 & 90 & 100 \\
Distance Modulus $(\Delta V, \mathrm{mag})$ & $2-10$ & 9.7 & 9.0 & 7.8 & 4.7 & 5.1 \\
\hline
\end{tabular}

stars to determine parameters such as age which are critical to understanding planetary evolution.

\subsection{TESS}

NASA has selected the Transit Exoplanet Sky Survey (TESS) for Phase A assessment as a candidate for SMEX launch around 2012-2015. Operating in equatorial, Low Earth Orbit, TESS utilizes 6 CCD cameras each viewing $18^{\circ} \times 18^{\circ}$ to build up a complete sky survey covering $2.5 \times 10^{6}$ bright host stars, with a precision 10-50 times greater than possible with ground-based surveys. The 1600 planets found in a TESS simulation include: 1100 gas giants, 400 Neptunes, 90 super Earths and 20 Earths.

\subsection{ASTRO}

A second concept for an all-sky survey is the All Sky Transit Observer (ASTrO; Beichman, PI) which uses 24 near-infrared cameras, each viewing $20^{\circ} \times 20^{\circ}$, to observe $24 \%$ of entire celestial sphere at one time with nearly $100 \%$ duty scale for periods of 60 days at low ecliptic latitude and up to 365 days at high ecliptic latitudes. As the spacecraft's orbit at L2 precesses at one degree per day throughout the year, ASTrO eventually covers the entire sky four times over the course of a four year mission observing more than $2 \times 10^{6}$ stars in unconfused sky $(\Omega>25,000$ sq. deg.) The near-IR operation $(0.66-1.65 \mu \mathrm{m})$ of ASTrO provides a factor of 2 or more SNR advantage over a CCD-based mission which translates into roughly $2^{1.5} \sim 3$ times as many $\mathrm{M}$ stars being observed. ASTrO's yield is over 2,000 planets, including 600 rocky planets transiting stars between 50 pc (M stars) and 200 pc (F stars) away (Figure 1).

While some of the all-sky goals of TESS and ASTrO could be achieved with networks of ground-based telescopes, a space mission with a 50-fold increase in sensitivity (particularly in the near-IR, Table 2) as well as favorable cadence and duty cycle, will be needed to detect the smallest planets around the closest (and reddest) stars.

\section{Future Follow-up Opportunities}

\subsection{HST}

Almost immediately after the discovery of the first transiting planets, HST demonstrated its power for transit research with abundance measurements of sodium in HD 209458b (Charbonneau et al. 2002) as well as evidence for an evaporating hydrogen exo-atmosphere (Vidal-Madjar et al. 2003; Ehrenreich et al. 2008). The loss of STIS removed this observational capability, but recent NICMOS grism observations have identified molecules in the atmosphere of HD 189733b (Swain et al. 2008) and Tinetti \& Beaulieu in this volume. Future NICMOS and ACS results in the visible and near-IR as 

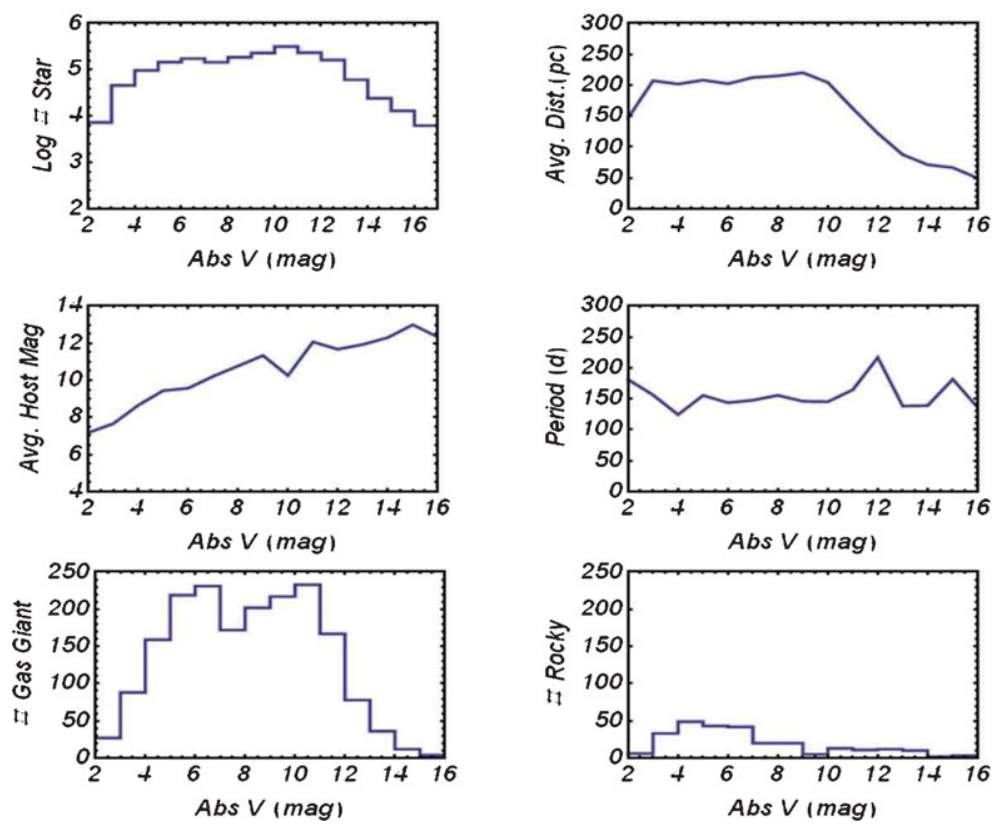

Figure 1. The ASTrO near-IR sky survey would find over 2,000 planets including more than 600 rocky planets, depending on assumptions as to the frequency of such objects. Various survey properties are shown as a function of absolute stellar magnitude of the host stars from A3 $\left(\mathrm{M}_{V}=2\right)$ to $\mathrm{M} 6\left(\mathrm{M}_{V}=16\right)$. The CCD-based TESS survey would have a similar yield but with about a factor of 3 fewer $M$ stars.

well as the Cosmic Origins Spectrometer and a resuscitated STIS spectrometer in the ultraviolet should greatly expand our knowledge of exoplanet atmospheres.

\subsection{Warm Spitzer}

Many contributions to this volume highlight the contributions of all three instruments on the Spitzer Space Telescope. From precision measurements of light curves to studying the time evolution of the emission from the atmosphere of a highly eccentric (and occulted!) planet (G. Laughlin, priv. comm.) to the presence or absence of a temperature inversion, Spitzer has helped to make the study of exoplanets into a science not a distant dream. Even after the cryogenic mission ends, the availability of thousands of hours of IRAC measurements of transits and complete light curves promises to be a critical adjunct to NASA's exoplanet program.

\subsection{JWST}

As the Cold Spitzer mission comes to a close and after the limited capabilities of Warm Spitzer are fully exploited, we will turn to James Webb Space Telescope (JWST) for follow-on observations of transits and full light curves of suitable exoplanets. In this section, I address briefly the capabilities of NIRSpec and MIRI which have been discussed elsewhere. I spend more time discussing NIRCam (Rieke et al. 2003) which has received relatively little attention so far in the exoplanet community.

\section{NIRSpec}

A number of authors, e.g. Clampin (2008), have highlighted the use of NIRSpec on JWST for transit observations with simulated observations showing resolved spectra lines of $\mathrm{H}_{2} \mathrm{O}$ and $\mathrm{CO}_{2}$ in the $1-2.5 \mu \mathrm{m}$ region (Valenti et al. 2005). The instrument mode of 

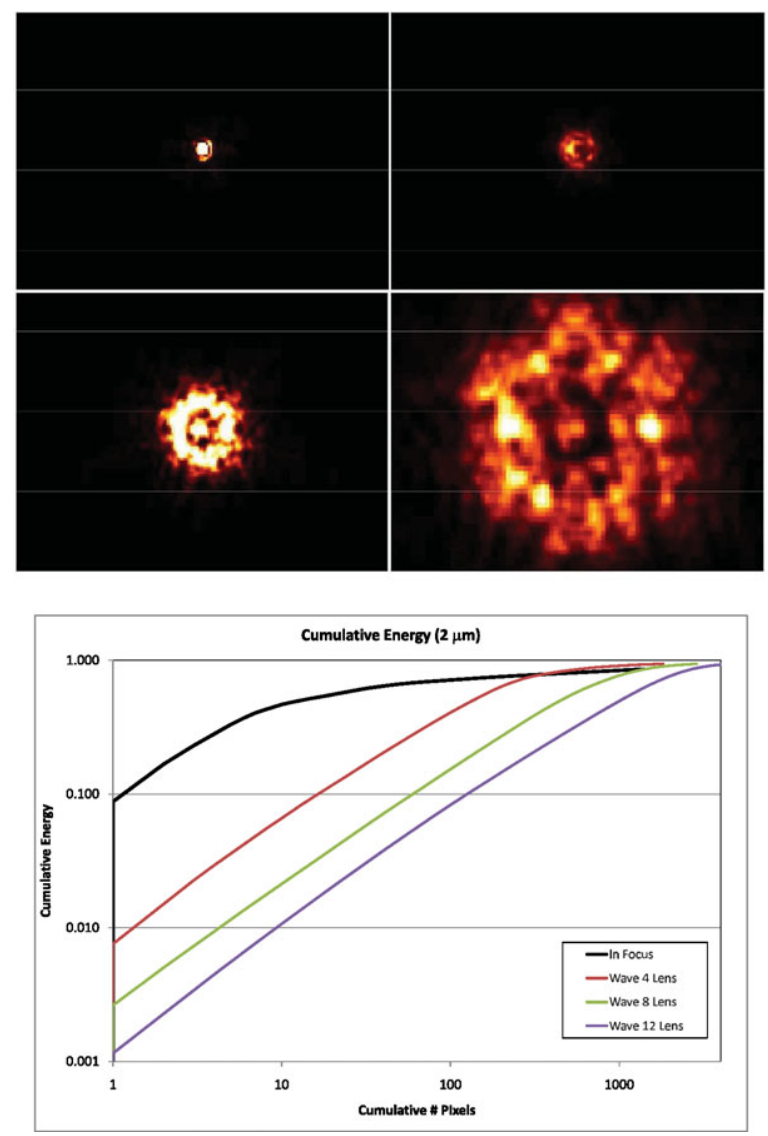

Figure 2. (top) The Point Spread Functions (PSF) at $2 \mu \mathrm{m}$ for in-focus plus 4, 8, $12 \lambda$ of defocus; (bottom) the cumulative energy within a given number of pixels: in-focus, 4, 8, $12 \lambda$ of defocus.

choice for these observations will be the fixed slit with either the prism $(\mathrm{R} \sim 35-100)$ or grating spectrometer $(\mathrm{R} \sim 1000)$ from $0.6-5.0 \mu \mathrm{m}$; scattered light from bright stars will probably make the multi-shutter array mode less desirable. An issue that will need to be addressed carefully is signal variability due to the coupling between pointing drifts and the losses in the $0.3^{\prime \prime} \times 4^{\prime \prime}$ slit. Flat-field errors resulting from NIRSpec's relatively coarse spatial sampling (100 mas/pixel compared to the diffraction-limited $\sim 100$ mas spot at 1-2 $\mu \mathrm{m}$ ) will also be an issue for the high dynamic range, high stability required for transit work. Averaging over many pixels will reduce this problem at the cost of lowered spectral resolution.

\section{NIRCam}

The weak lenses (short-wavelength channel) and grisms (long wavelength channel) recently added to NIRCam for the initial capture and long term alignment of the JWST telescope will make a dramatic improvement in NIRCam's capabilities for transit measurements. The short- $\lambda$ pupil wheel contains two weak lenses which introduce $\pm 8 \lambda$ of defocus. These can be used individually with any of the filters in the filter wheel or in combination with another weak lens in the pupil wheel which introduces a defocus of $+4 \lambda$. A total defocus of $+12 \lambda$ is thus possible, although the $4 \lambda$ lens has an integral F212N coating $(\mathrm{R} \sim 100)$ making this combination not generally useful. 


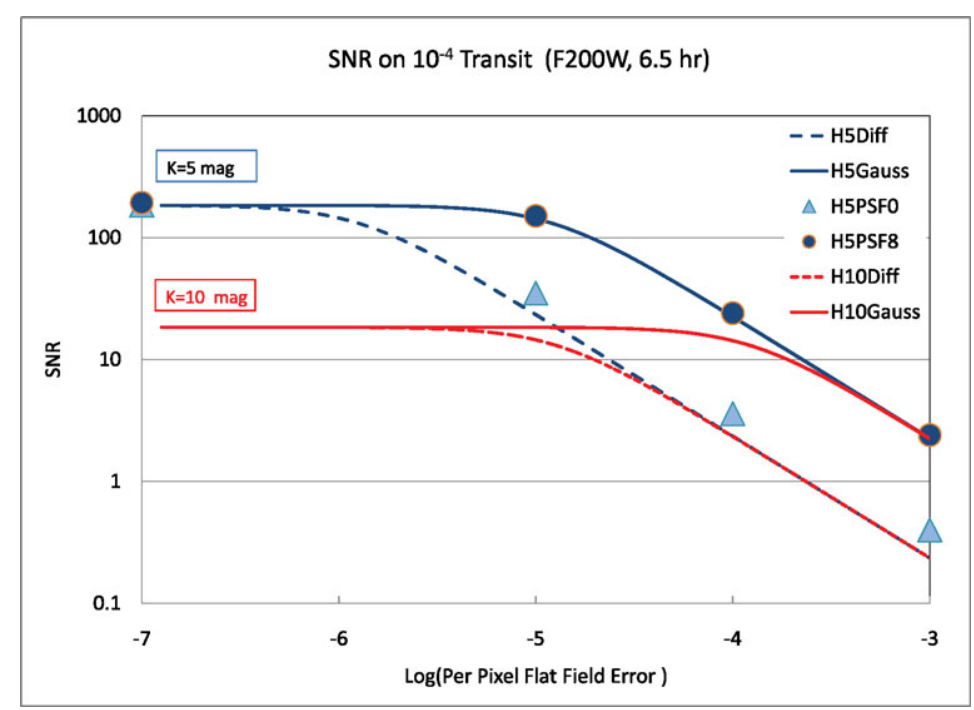

Figure 3. The two upper curves show the SNR of an earth in front of a $\mathrm{K}=5 \mathrm{mag} \mathrm{G} 2 \mathrm{~V}$ star for diffraction limited (blue, dashed) and Gaussian FWHM=30 pixel (blue, solid) cases as a function of the per-pixel flat field noise parameter (Eqn. 3). The lower pair of curves (red) represent the same cases for a fainter, $K=10 \mathrm{mag}$ star. The filled symbols represent calculation of SNR using realsitic PSFs for a $K=5$ mag star in the diffraction limited case (blue triangles) and for diffused light using the $8 \lambda$ weak lens (blue circles).

In the case where "flat field" noise due to imperfect flat-fielding, pointing drifts interacting with inter- and intra-pixel variations, etc., dominates, spreading the light from the star over many pixels is essential. The power of pixel-averaging was dramatically demonstrated in the HST/STIS spectrum of HD 209458b where a light curve with SNR $>10^{4}$ was obtained by averaging over more than 1,000 pixels (Brown et al. 2001).

The reduction in the "flat field" noise obtained by averaging over a large number of pixels is shown in Figure 3 which gives the SNR for a transit of depth $10^{-4}$, i.e. an Earth in front of a G2 star, as observed by JWST observing at a star with [F200W] $=5$ or 10 mag for typical transit length, $6.5 \mathrm{hr}$. The curves show results for a diffraction limited and Gaussian beams (FWHM=30 pixels) and various values of the "flat field" parameter, $f$. The "flat-field" noise completely dominates the shot noise for the bright star. Using the predicted JWST PSF (Krist 2007) we can address the effect of flat-field noise more realistically. Figure 2a shows an in-focus, JWST image at $2 \mu \mathrm{m}$, as well as images with 4, 8, $12 \lambda$ of defocus. Cumulative energy curves (Figure 2b) show that with the $8 \lambda$ weak lens over half of the starlight is spread over 500 pixels compared with just 15 pixels at best focus. SNR calculations (symbols in Figure 3) using realistic PSFs agree well with the predictions of the model.

Using the weak lenses, NIRCam will be able to obtain highly precise light curves in most of the NIRCam filter set for improved estimates of planetary and stellar properties, timing variations due to other planets, and searches for rings or moons. The most popular filter may be the F150W2 with a ultra-wide passband from 1-2 $\mu \mathrm{m}$ which will allow the highest possible SNR. With the averaging of per pixel variations, JWST should achieve the expected $D_{J W S T} / D_{K \text { epler }}>6$-fold improvement in SNR compared with Kepler, making this mode very attractive for the follow-up of the faint transit signals from terrestrial planets. 


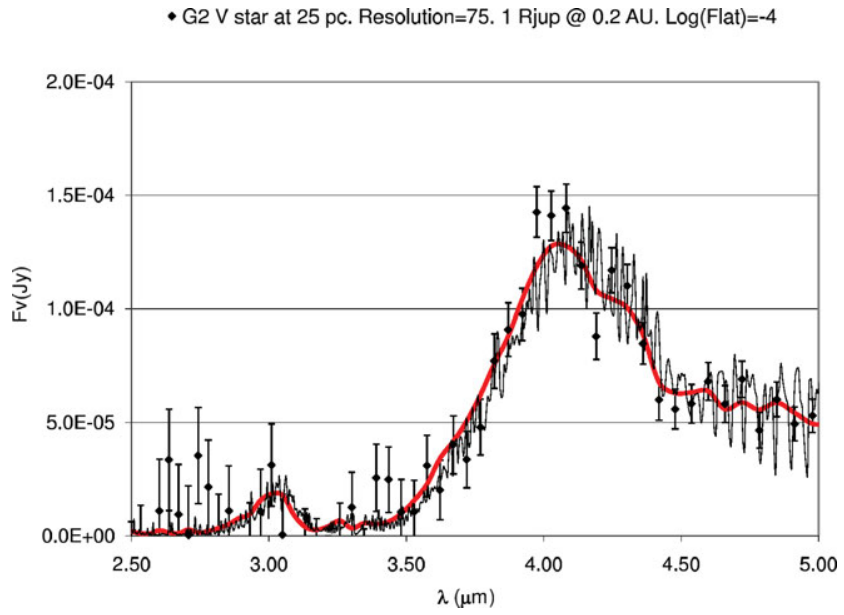

Figure 4. A simulated NIRCam grism spectrum of a hot Jupiter transiting a G2 star based on a model for the day-side of TrES-1 (Burrows et al. 2008). The $\mathrm{R}=75$ smoothed spectrum is shown in red, the full resolution model as faint black line. Data with error bars are for $6.5 \mathrm{hr}$ of integration with "flat-field" errors of $10^{-4}$ averaged over 200 pixels, as appropriate for this spectral resolution.

\section{Grism Observations with NIRCam}

Numerous observational and theoretical contributions to this volume show that much can be learned about atmospheric composition and structure from spectroscopy in the 1-5 $\mu \mathrm{m}$ band. The long wavelength grism of NIRCam will probe wavelengths $(2.4-5 \mu \mathrm{m})$ not observed by either Spitzer or HST (Greene et al. 2007). This is a very rich area of the spectrum (Figure 4, Burrows et al. 2008). Observing in secondary eclipse (emission) JWST would be able to measure a hot Jupiter going behind a $[4.5 \mu \mathrm{m}]=5$ mag G star with $\mathrm{SNR}=10 \sim 20$ at a resolution 500 in just 1 hour (depending on the assumed value of the flat field error). Viewed in front of an M3 V star, the corresponding SNR would be $\sim 200$ per spectral pixel! For a hot "super-Earth" $\left(2 \mathrm{R}_{\oplus}\right)$ transiting behind an M3V [4.5 $\mu \mathrm{m}]=5 \mathrm{mag}$ star, it would be possible to achieve an SNR of 15 at a resolution of 50 . For more distant, "cold" planets seen in primary eclipse, JWST can achieve SNR of many tens to hundreds for Jupiters in front of M-G stars at resolutions of 500. Super-Earths can be observed spectroscopically with $\mathrm{SNR} \sim 5-10$ at resolutions of 50 .

Important advantages of the NIRCam grism include its slitless operation which means an absence of time-variable slit losses that presently limit the sensitivity of Spitzer/IRS observations (Swain et al. 2008a) and which could similarly affect JWST/NIRSpec. In addition, the grism can operate in parallel with both: a) the short wavelength module which can be obtaining a light curve at some other wavelength; and b) the long and short wavelength modules in the other arm of NIRCAM which can be monitoring pointing and detector drifts on a different patch of sky. Finally, it is important to note that discussions are underway to identify a short wavelength grism that could replace a short-wavelength filter to provide these same benefits for the 0.6-2.4 $\mu \mathrm{m}$ range of NIRCam. A short wavelength grism is also under discussion for the Canadian Tunable Filter instrument (Doyon, private communication).

\section{Detector Tests}

Experiments are underway at the University of Arizona to make high-precision flat-field measurements to measure flat-field uncertainties, and to test techniques for the removal 


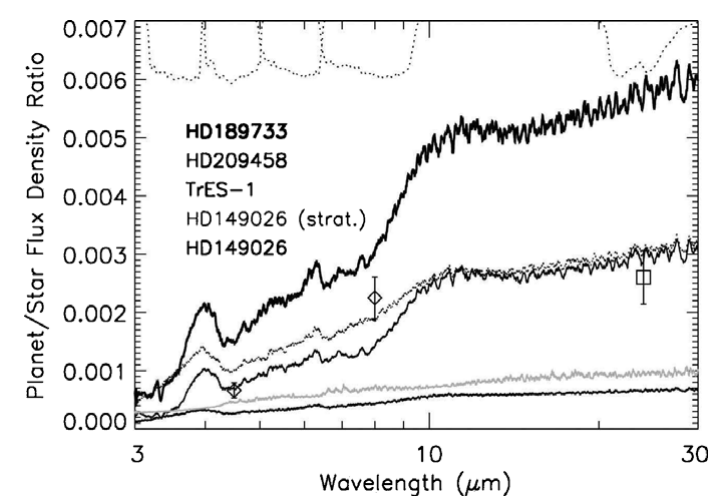

Figure 5. Theoretical (continuous) and observed (points) planet-to-star flux density ratios for HD 189733, HD 20458, TrES-1, and HD149026 with and without a stratsophere from top to bottom, reproduced from Fortney et al. (2006). Strong $\mathrm{H}_{2} \mathrm{O}$ and $\mathrm{CH}_{4}$ absorptions in the $\lambda=6-10 \mu \mathrm{m}$ range constrain effective temperatures and abundances.

of detector drifts (red noise). Initial tests suggest flat field error of $<2.5 \times 10^{-4}$ over $0.5 \mathrm{hr}$. Tests with hundreds of full well frames will be used to understand the stability of the NIRCam detectors for transit measurements.

\section{What is the Brightest Star that JWST Could Observe?}

An advantage of the NIRCam arrays is their ability for fast readout of a small window on the array. In this mode integration times of a tenth of a second or less are possible. Observing with the $12 \lambda$ lens with its integral F212N filter would result in no more than $\sim 1 \times 10^{5}$ electrons (roughly full-well) in the brightest pixel for a $\mathrm{K}=1 \mathrm{mag}$ star in $0.25 \mathrm{sec}$ $(160 \times 160$ pixels $)$ and would yield a high quality light curve for an Earth/G2V transit. In grism mode, a resolution of $\mathrm{R}=1500$ results in no more than $1 \times 10^{5}$ elec in $0.16 \mathrm{~s}$ $(1024 \times 16$ pixels with 10 pixel spot) for a $[4.5 \mu \mathrm{m}]=3 \mathrm{mag}$ star $(\mathrm{G} 2 \mathrm{~V}$ star at $\sim 7 \mathrm{pc})$.

\section{MIRI}

Primary transit and secondary eclipse observations in the mid-IR are very useful for studying both giant and terrestrial exoplanets. Very strong $\mathrm{H}_{2} \mathrm{O}$ and $\mathrm{CH}_{4}$ absorptions in the $\lambda=6-10 \mu \mathrm{m}$ range constrain the temperatures and abundances in the atmospheres of warm giant planets (Figure 5). Low resolution mid-IR spectra of terrestrial planets may show continuum emission from their surfaces with superposed absorptions of $\mathrm{H}_{2} \mathrm{O}$ $(8 \mu \mathrm{m}), \mathrm{O}_{3}(9 \mu \mathrm{m})$, or $\mathrm{CO}_{2}$.

The JWST mid-infrared instrument (MIRI) will operate over the $\lambda=5-28 \mu \mathrm{m}$ region with a multi-filter camera and a medium resolution $(R \sim 3000)$ integral field spectrograph (MRS; Wright et al. 2004). The MIRI low resolution $(R \sim 100)$ double-prism spectrograph (LRS) will operate over the $\lambda=5-14 \mu \mathrm{m}$ region. The camera and low resolution spectrograph have pixels that map to 110 mas on the sky, providing Nyquist sampling of the JWST PSF at about $7 \mu \mathrm{m}$ wavelength; the IFU images are generally undersampled. It is possible to operate the MIRI LRS in slitless mode much like the NIRCam grisms, and this configuration is likely to yield the highest quality observations of exoplanet primary transits, secondary eclipses, and light curves. MIRI long-wavelength ( $\lambda \sim 20 \mu \mathrm{m})$ imaging observations will also be oversampled and will likely yield precise photometry. MIRI data will be very powerful for studying cooler transiting planets, and combining MIRI emission spectra with NIRCam transmission spectra will likely reveal much about the atmospheric temperatures, constituents, and profiles of transiting exoplanets. 
Warm planets will have higher signal-to-noise secondary eclipse spectra in the mid-IR than the near IR. A T=900 K planet orbiting 0.1 AU from a G2V star would have an orbital period of approximately 12 days. The thermal emission from this planet could be observed 3 times faster at $\lambda=10 \mu \mathrm{m}$ than $\lambda=3 \mu \mathrm{m}$ at the same signal-to-noise. If this system were at $10 \mathrm{pc}$ distance, a high signal-to-noise $(\sim 50)$ spectrum could be obtained for a Jupiter-sized planet with the MIRI LRS in about $3.0 \mathrm{hr}$ total, within a single secondary eclipse period $(1.5 \mathrm{hr})$. Cooler, longer period planets are likely to be discovered with space-based transit surveys and should have dramatically better data quality in the mid-IR than the near-IR. A Jupiter-sized planet identical to the one above but orbiting a similar star at a distance of $0.2 \mathrm{AU}$ would have a 33-day period and a blackbody temperature of $630 \mathrm{~K}$. Approximately 15 hours of observing time would be needed to obtain a $\mathrm{S} / \mathrm{N}=50$ spectrum of the planet with MIRI LRS at $\mathrm{R}=100$, which could be obtained over only a few periods ( $7.5 \mathrm{hr}$ integration time in secondary eclipse).

Perhaps the most exciting aspect of MIRI is that it will likely be able to observe the thermal emission of terrestrial exoplanets. A 2 Earth-radius planet orbiting an M3 dwarf at a distance of $0.1 \mathrm{AU}$ would have a 21 day transit period. This planet would have a blackbody temperature of $320 \mathrm{~K}$ and would be in the star's habitable zone. If the planet were 10 Earth masses, then it would induce a $5 \mathrm{~m} \mathrm{~s}^{-1}$ radial velocity signal on its star. JWST MIRI could detect the thermal emission signal at $\mathrm{S} / \mathrm{N}=10$ from this planet in only 6 hours total integration time in its broad-band 2100W filter if the system were 5 pc distant; this planet could be detected with an observation spanning a single secondary eclipse. Smaller planets could be detected by co-adding multiple data sets.

\section{Other Follow-on Space Opportunities}

- EPOCH/EPOXI (Deming, PI). An indication of the excitement of IAU Symposium 253 was the daily updating of the light curve of GJ436 obtained with the $30 \mathrm{~cm}$ telescope on the Deep Impact spacecraft (Chistiansen et al., this volume). The EPOCH project is monitoring at least 4 stars in the visible looking for secondary transits in reflected light, evidence of rings and moons, co-planar planets of smaller (terrestrial) radius, or timing variations due to other planets. It is worth noting that the out-of-focus camera that was an unfortunate characteristic of the prime mission has proven to be a blessing for the high precision photometry needed for transit photometry.

- TRACER (Clampin, PI; Charbonneau, Deputy PI). is a SMEX-class proposal to use a 0.6 meter telescope with a two channel spectrograph $(0.8-1.7 \mu \mathrm{m}, \mathrm{R} \sim 50)$ to characterize atmospheric properties (physical conditions, chemistry, dynamics and cloud properties); determine physical structure (precision light curves for robust radii, detection of rings and/or satellites); and detect unseen planets, e.g. co-planar, terrestrial planets in transit or via perturbations of transit timings.

- THESIS (Terrestrial and Habitable-zone Exoplanet Spectroscopy Infrared Spacecraft; Swain, PI) is a concept to utilize a $1.5 \mathrm{~m}$ telescope optimized for 2 - $15 \mu \mathrm{m}$, $\mathrm{R} \sim 500$ spectroscopy using molecules as probes of conditions, composition, and chemistry. THESIS's science goals include non-transiting exoplanet spectroscopy; day and night characterization of $\mathrm{M}$ dwarf planets in the habitable zone; full orbit spectroscopic light curves; long-term weather monitoring; and high-precision transit timing.

\section{Conclusions}

The era of planet characterization is now upon us, at least for "Hot Jupiters" with an extension to cooler, small planets in the offing with the advent of the CoRoT and Kepler 
surveys, the Spitzer Warm Mission, refurbished HST and ultimately all three instruments on JWST. There remain, however, some missing pieces in the program. First, we would like to identify the smallest possible planets around the closest, brightest stars and expand the transit census to include M stars. This will require an all-sky survey in the deep-red or near-IR from space for ultimate sensitivity. The selection of the TESS mission for a Phase A study is a welcome step in this direction. Ultimately, however, we would like to make a still more dramatic step, namely survey the closest stars for terrestrial planets, not relying on the chance alignments that make transits possible. An initial astrometric survey and characterization with the Space Interferometer Mission (SIMLite, Beichman et al. 2008) could be followed by dedicated characterization missions at visible and infrared wavelengths with TPF-C and TPF-I/Darwin (Beichman et al. 2007). Until such time as these long-term goals can be achieved, the observations of transiting planets from space will yield a wealth of information that will greatly advance our understanding of individual planets.

\section{Acknowledgments}

The research described in this paper was carried out at the Jet Propulsion Laboratory (JPL), California Institute of Technology, under contract with the National Aeronautics and Space Administration. Work with NIRCam at JPL is supported under JPL Contract Task Plan No. 70-7920.

\section{References}

Beichman, C. A., et al., in Protostars and Planets V, B. Reipurth, D. Jewitt, and K. Keil (eds.), University of Arizona Press, Tucson, p. 915.

Beichman, C. A., et al. 2008, in Proceedings of IAU 248, in press.

Brown, T. M., Charbonneau, D., Gilliland, R. L., Noyes, R. W., \& Burrows, A. 2001, ApJ, 552, 699.

Burrows, A., Budaj, J., \& Hubeny, I. 2008, ApJ, 678, 1436.

Clampin, M. 2008, Advances in Space Research, 41, 1991.

Charbonneau, D., Brown, T. M., Noyes, R. W., \& Gilliland, R. L. 2002, ApJ, 568, 377.

Ehrenreich, D., et al. 2008, A \& A, 483, 933.

Fortney, J. J., Saumon, D., Marley, M. S., Lodders, K., \& Freedman, R. S. 2006, ApJ, 642, 495.

Green, D., Matthews, J., Seager, S., \& Kuschnig, R. 2003, ApJ, 597, 590.

Greene, T. 2007, in Proceedings of SPIE, Techniques and Instrumentation for Detection of Exoplanets III, Daniel R. Coulter, Editor, Vol. 6693, in press.

Krist, J. 2007, in In the Spirit of Bernard Lyot, UC Berkeley. Ed. P. Kalas.

Rieke, M., et al. 2003, in IR Space Telescopes and Instruments. Ed. J. C. Mather, Proc. SPIE, $4850,478$.

Roxburgh, I. \& Catala, C., Plato Consortium 2007, Communications in Astroseismology 150, 357.

Swain, M. R., Bouwman, J., Akeson, R. L., Lawler, S., \& Beichman, C. A., ApJ, 674, 482.

Swain, M. R., Vasisht, G., \& Tinetti, G. 2008, Nature, 452, 329.

Valenti, J., et al. 2005, BAAS, \#115. 05, 37, 1350.

Vidal-Madjar, A., Lecavelier des Etangs, A., Désert, J.-M., Ballester, G. E., Ferlet, R., Hébrard, G., \& Mayor, M. 2003, Nature, 422, 143.

Wright, G. S., et al. 2004, Proc. SPIE, 5487, 653. 